\title{
A method to analyze the spatial distribution of behavior
}

\author{
ELIAS ROBLES \\ Centro de Investigación en Alimentación y Desarrollo, A.C., Hermosillo, Sonora, Mexico
}

\begin{abstract}
This article presents a method comprising an experimental environment, infrared detection system, data collection and processing software, behavior categories, and suggested quantitative analysis methods for studying some spatial properties of behavior. In the chamber, the location of a rat is determined at regular intervals by a $24 \times 24$ grid of infrared beams; the status of each line used interactively to determine changes in the environment are stored on disk for later analysis. From the status of the receptors, the computer decodes the location of objects in the chamber, and a binary 576-cell matrix is obtained for each sample. The series of matrices allows a number of behavior categories to be defined including exploration, activity, and time allocation. Exploration curves and time allocation surfaces are compared using a goodness-of-fit test; activity is assessed with time-series methods. Potential applications to psychological, pharmacological, and ethological research are considered.
\end{abstract}

A number of important phenomena regarding the spatial distribution of behavior, including general motor activity, exploration, and choice of location, have been studied as a function of a diverse set of physical, social, and physiological variables. Methods developed to operationally define and measure exploration include the openfield (Hall, 1934), the hole (Krnjevic \& Videk, 1967), and the tunnel tests (Shillito, 1970). Similarly, the running wheel (Strong, 1957), the stabilimeter (Campbell, 1954), the capacitance change detector (Siegel, 1946), the photocell detector (Dews, 1953), and the infrared beam detector (Silverman, Chang, \& Russell, 1988; Ulett, Heusler, \& Callahan, 1961) were designed to study general activity and/or translation. The video digitizer detector (Livesey \& Leppard, 1981; Pear \& Eldridge, 1984) and numerous types of mazes (Barnett \& Smart, 1970; Lyons \& Cheney, 1984; Staddon \& Ayers, 1975)--some using microswitches as sensing devices-are used to study time allocation in space.

Although activity, exploration, and time allocation in space are closely interrelated, for the most part they have been studied separately (Shillito, 1970). Undoubtedly, theoretical considerations gave rise to this diversity, but the technologies involved, although relatively successful in measuring the specific phenomena for which they were designed, have not facilitated integration of the results. With the exception of the video digitizer, the devices used to measure one of these behaviors do not provide data to assess the others.

This article was based on a dissertation submitted by the author to the Department of Psychology, University of Arizona, in partial fulfillment of the requirements for a $\mathrm{PhD}$. Requests for reprints and program files may be sent to Elias Robles, CIAD, Apartado Postal 1735, Hermosillo, Sonora, Mexico. Send one blank, formatted 360K diskette and a self-addressed pack to receive the program listings.
Comparative analyses have demonstrated the adequacy of optical devices, such as photocell and infrared detectors, to assess activity (Strong, 1957; Weasner, Finger, \& Reid, 1960). Optical devices seem to be more sensitive and reliable than do mechanical devices, such as running wheels and stabilimeters, when measuring activity in rats and mice. Infrared technology has been used successfully in the study of the behavior of insects (Brown \& Unwin, 1961; Miller, 1979). However, the spatial resolutions required by most species of invertebrates, and by such behaviors as wall climbing and flight, favor the use of video technology. Also, in environments such as the water maze, where the measurement of the subject above objects along the horizontal plane of the detectors cannot be assured, the use of infrared technology is not practical. On the other hand, infrared devices are immune to false positives produced by video detectors when contrasting objects, such as food, wood particles, or feces, are deposited on the chamber's floor. Therefore, when the spatial location of rodents is to be studied in the laboratory or in limited natural settings, infrared technology provides an inexpensive and reliable alternative to video digitizers. This is specially true when such global measures as exploration, amount of translation, or choice of location are targeted.

The purpose of this paper is to present an inexpensive and reliable method to define and analyze exploration, general activity, and time allocation in space, from a common set of spatial location data obtained with infrared technology.

\section{APPARATUS}

The system consists of a computer-controlled $24 \times 24$ matrix of infrared devices evenly arranged along the sides of a square box. Every $n$ seconds, a reading is made and 
a binary value is obtained that describes the status of each of the 48 beam receptors. A 0 is assigned to the beams that reach the receptor on the other side of the box, and a 1 is assigned to the beams that do not. From this string, composed of 24 digits for the $x$-axis and 24 digits for the $y$-axis, the computer decodes the coordinate pairs that describe the physical location of objects in the chamber. The basic 48-digit strings can be immediately decoded or sequentially stored on disk for further treatment. In turn, a set of computer programs process the data files by computing measures of motor activity, exploration, and time allocation.

\section{Experimental Chamber}

The chamber $(1.20 \mathrm{~m} \times 1.20 \mathrm{~m} \times 0.60 \mathrm{~m})$ was made of 1.27 -cm-thick sheets of plywood (see Figure 1). Underneath, two $(1.20 \mathrm{~m} \times 5.08 \mathrm{~cm} \times 5.08 \mathrm{~cm})$ wooden rails kept it raised from the floor. A row of 24 evenly spaced holes $(0.50 \mathrm{~cm}$ in diameter) was drilled in each wall $3.5 \mathrm{~cm}$ above the floor of the box to hold the optoelectronic devices. The interior walls and floor were lined with $0.63-\mathrm{cm}$-thick clear Plexiglas and sealed with silicone to prevent moisture from permeating the wood structure. The clear plastic allows infrared beams to travel freely across the box while keeping the optical devices inaccessible to the animal, and it permits thorough cleaning and disinfecting of the chamber. A $7.62 \mathrm{~cm} \times 12.70 \mathrm{~cm}$ wedge-shaped wire-mesh food hopper and a water bottle were attached to the center of the north wall 1 in. above the infrared devices. The floor of the box was covered with a thin layer of commercially available wood particles to absorb moisture from excretions.

\section{Detecting Circuits}

To control the status of the $\mathbf{4 8}$ infrared light-emitting diodes (IRLEDs), six 8-bit programmable input/output
(I/O) ports were used. The ports were purchased from Alpha Products (242-F West Ave., Darien, CT) and are built around Intel's 8255A chip, so three programmable ports are available in each card. Another two cards were used in the input circuits described below. All I/O port cards were attached to a proprietary bus connected to a controller card that was attached to the computer's motherboard. The bus is provided with its own power supply; the controller card draws power directly from the computer. An adapter card, cable, motherboard, and four 24-line I/O cards cost about $\$ 500$.

Each IRLED (SEP8703-1, Radio Shack Part No. 276143 ) is independently excited as selected by the output port lines (see Figure 2). The 40-kHz pulse train generated by a 555 timer is required to set off the infrared detector (GPIU52X, Radio Shack Part No. 276-127). Therefore, the output of the free-running oscillator is AND-gated ( $1 / 47408)$ with each of the 48 output lines, and the resulting signals are used to excite the IRLEDs while that particular output line is high.

On the input side, the signal coming from each detector is connected directly to each input port line. This simple arrangement, then, requires the computer to sequentially excite each IRLED, read the status of the detector, and turn off the IRLED. Although this keeps the processor busy for a while, sequentially reading one line at a time makes it possible to obtain accurate records at relatively high density - even when the distance between the LEDs and the receptors is over $2.0 \mathrm{~m}$-without having to use collimating lenses and precise mechanics. The cost of 48 IRLEDs and IR detectors is about \$250; supporting circuitry and hardware cost about $\$ 50$.

\section{Computer}

A Tandy 1000 personal computer was used to run the experimental sessions. It is an IBM-compatible system

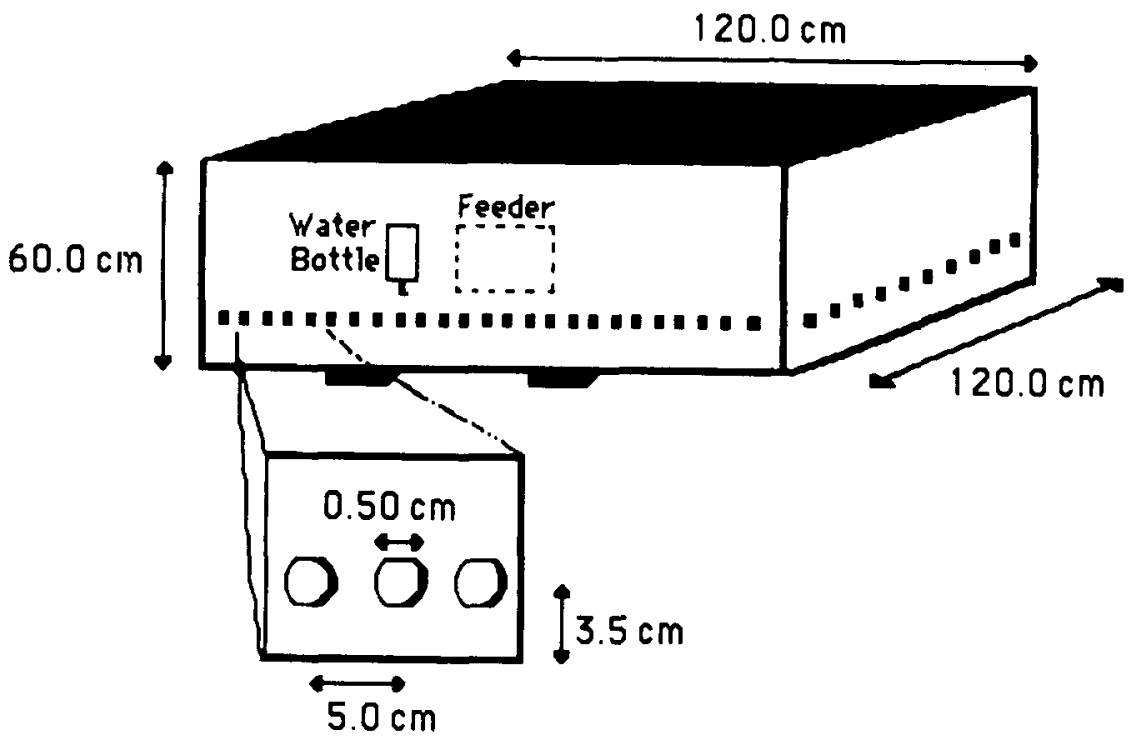

Figure 1. Diagram of the experimental chamber. 


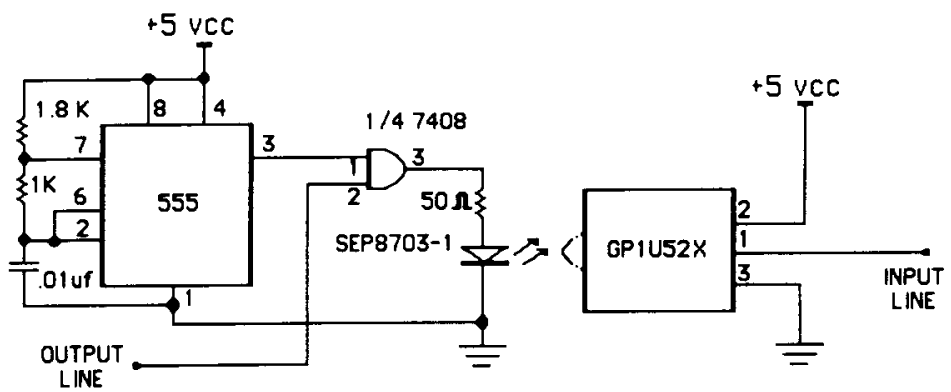

Figure 2. Diagram of the infrared detection circuits. Twenty-four LED-detector pairs were used for the $x$-axis and 24 for the $y$-axis; oscillators were common to 12 such pairs.

based on the 8088 microprocessor running at $4.77 \mathrm{MHz}$ under the MS-DOS (release 3.10) operating system, with 640K random access memory (RAM), 20MB hard disk, and $360 \mathrm{~K}$ disk drive. The integrated video subsystem was a color graphics adapter (CGA), with a monochrome composite video monitor.

Since a sample was taken every $5 \mathrm{sec}, 17,280$ readings were obtained daily. In 1 week, such a number of readings creates a data file over 6 million bytes long, which would fill over $15360 \mathrm{~K}$ diskettes. Alternatively, it is possible to store each sample as two three-digit integers in order to save storage space. However, storing the 48-digit strings allows one to quickly browse through the file and identify specific passages of an experimental session, without having to decode this information. Therefore, to circumvent the problems of storage space and portability, a relatively fast, commercially available data compression package, PKARC (PKWARE, Inc., 7032 Ardara Ave., Glendale, WI), was used to reduce the size of the ASCII files created by about $96 \%$, thus making it possible to hold all data files for each subject on a single $360 \mathrm{~K}$ diskette.

\section{Software}

Computer programs were written to run the sessions and create data files, as well as to analyze and graphically represent the data. The code was written in Turbo Basic v1.0 (Borland International) but can be readily implemented in other BASICs (e.g., Microsoft's QuickBASIC).

Listing 1 presents a listing of BBSCAN.BAS, the program designed to run experimental sessions. It consists of four subroutines to declare variables and addresses, scan, control the flow, and finish the program. Briefly, upon entry to the program, subroutine MAIN is executed. This routine first directs the program flow to subroutine SETUP, which performs a number of preliminary functions. It establishes a keyboard control to abort the session, defines arrays, defines ports as either input or output, declares $\mathrm{I} / \mathrm{O}$ port addresses (these may vary depending on the particular computer used, check your technical reference manual for available ports), opens the data file buffer, computes the length of the session, resets the timer, and initiates the session. This routine also accepts from the user the name of the output file and the length of the session in days, hours, minutes, and seconds. Upon returning to subroutine MAIN, a software interrupt is enabled that executes subroutine SCAN every $5 \mathrm{sec}$ until $N$, the number of readings made, matches the session length. When either the keyboard interrupt is activated or the session is over, subroutine WAYOUT is executed. This segment of the program resets the ports, disables the timer, closes the data file, and signals the end of the session. Finally, subroutine SCAN writes and reads the status of each of the six input ports eight times, masking on every occasion the value of only 1 bit, creates a string for $x$ and another one for $y$, and writes the resulting 24-digit strings to both the screen and the data file.

\section{Time Allocation}

When the relative frequency of occupying one of the 576 possible cells is computed over a large number of samples, a data matrix is obtained that describes time allocation in space. Collapsing the data into such a matrix generates a global measure that can be used in studies of "preference," in which ethologically relevant behavior (e.g., nesting, foraging, and patrolling) can be quantitatively described as particular instances. The function of program PREFERNS.BAS (see Listing 2) is to create the data file from which surface charts are generated to depict relative frequency counts in each cell in the box. Briefly, the program consists of four routines, which initiate files and variables, decode spatial location, write the data file, and end the program. Subroutine SETUP defines a $24 \times 24$ element array to hold absolute frequencies, opens the I/O files, and sets a software interrupt to abort the program. Next, routine DECODE identifies the $x, y$ coordinates occupied by the animal in each reading and increments the count in the corresponding elements of array $\mathrm{M}$ accordingly. When all samples in the raw data file have been accounted for, subroutine GRAPH writes the relative frequency data file in ASCII format. Finally, the routine WAYOUT closes both files, signals the end of the program, and returns control to the operating system. Figure 3 shows a rat's time allocation surface obtained during a 24-h period. In this surface, the selected nest site and the food/water area can be easily identified. The following day, when an artificial nest was attached to the center of 


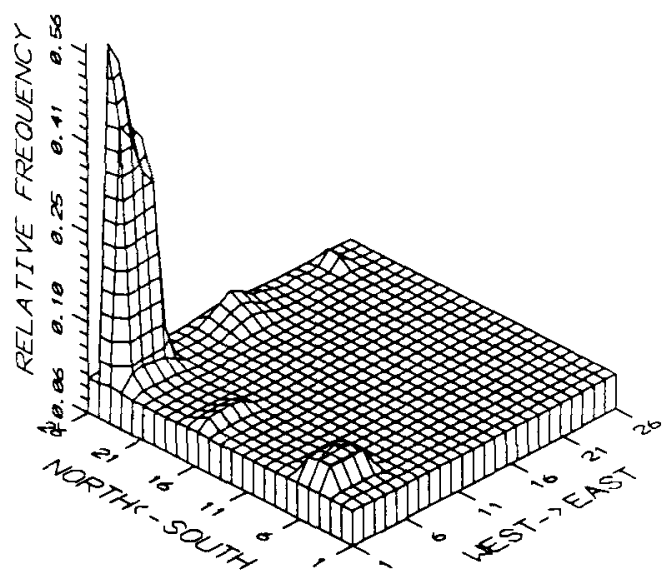

Figure 3. Choice of location as relative frequency of chamber cells occupied by a rat over 17,280 samples.

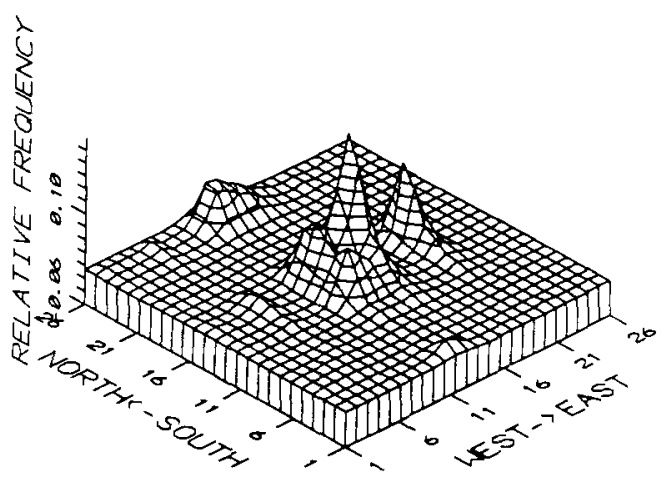

Figure 4. Relative frequency of occupied cells over 17,280 samples, with nesting material affixed to the center of the floor of the chamber.

the floor of the chamber, the distribution changed, as shown by Figure 4. The Kolmogorov-Smirnov goodnessof-fit test can be used to quantitatively compare time allocation data. By transforming the matrix into a vector, a unique distribution is obtained that can be compared with a theoretical distribution or with another equally transformed matrix (Siegel \& Castellan, 1988).

\section{Activity}

In most applications of optical technology, the IR beams are continuously present, and the number of times they are interrupted is counted as a measure of motor activity (see, e.g., Silverman, Chang, \& Russell, 1988). In the present case, the animal is always obstructing the path of several beams, and all receptors are periodically sampled to identify its location. Therefore, activity is defined in terms of the number of visited cells per unit of time, instead of the number of individual beam interruptions. Thus, this measure describes the rate of location change. Program ACTIVITY.BAS (see Listing 3) uses raw data from BBSCAN.BAS to create a file containing the percentage of the box covered by the animal in successive time in- tervals. The length of the intervals can theoretically vary from twice the sampling interval to the full duration of the session. This program is made up of three routines. Subroutine SETUP declares variables, starts software interrupt to abort the program, and gets names for and opens the I/O files. In turn, DECODE computes the $x, y$ coordinates from each reading, increments the respective frequency counts in array $M$, accumulates percentages in intervals (with size represented by variable CHUNK), and resets the content of array $\mathbf{M}$. Lastly, WAYOUT closes the I/O files, signals the end of the run, and stops the program. The data files generated by this program may be directly plotted or used to perform the time-series analyses. To assess the adequacy of this measure of activity, a rat's location was recorded during 15 continuous nights. It was determined a priori that an adequate base interval to describe activity in the rat would be $15 \mathrm{~min}$. Therefore, data from 180 samples were added to obtain each point. The data points corresponding to the first 14 nights were then used to generate a model using Box-Jenkins methods (Pankratz, 1983). Figure 5 depicts the 12 obtained and predicted data points corresponding to Observations 118 to 129 of Night 15 , as well as the points marking the $95 \%$ confidence intervals. The data show a consistent pattern of activity with a sharply marked 3-h cycle. Note how the forecasted points track the pattern in the series, following the extreme periodic undulations very well. All of the forecasted values fell within the estimated $95 \%$ confidence intervals.

\section{Exploration}

From the basic data matrix produced by this system, it is also possible to compute an index to describe exploration. By keeping track of the cumulative count of unique cells visited by a rat starting at a particular arbitrary time, a curve is generated that describes the percentage of the

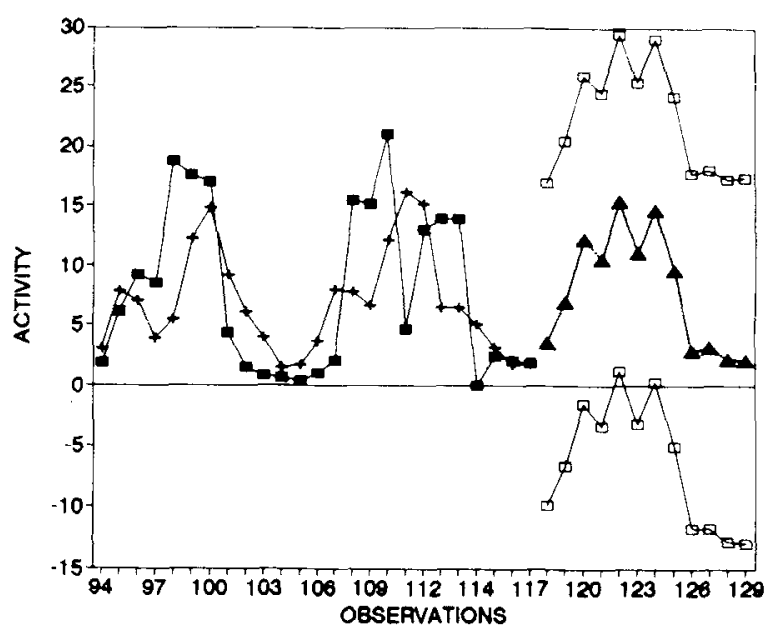

Figure 5. Activity of a rat during Night 15 in the experimental chamber. The chart shows actual data (filled squares) and the corresponding values forecasted at lead $(+)$, as well as forecasted values for Observations 94 to 129 (nlled triangles) and the corresponding 95\% confidence limits (open squares). 


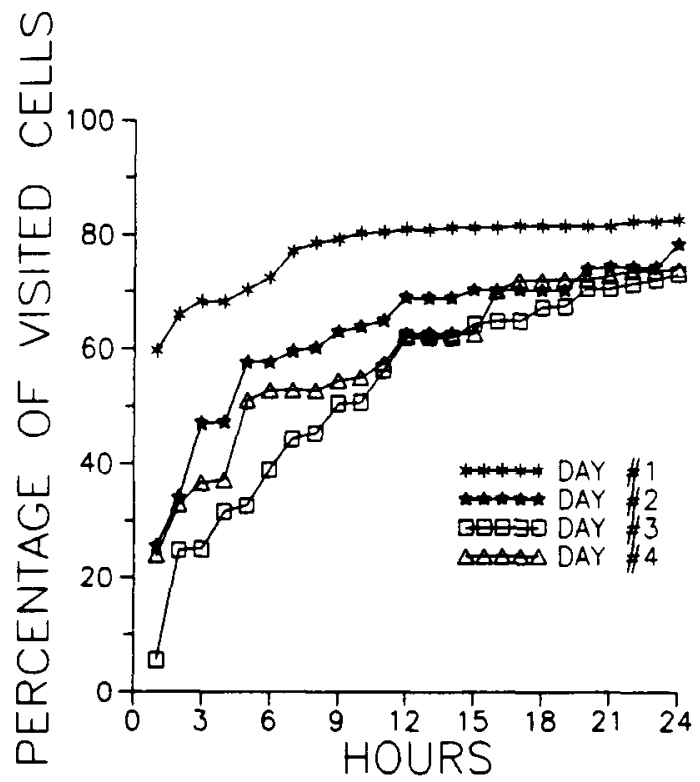

Figure 6. Exploration as cumulative percent of unique cells visited. The chart shows a rat's first 4 days in the chamber.

total area visited as a function of time. Figure 6 shows the hourly cumulative percentage of the total area visited by a rat during the first four days in the chamber. Data for the cumulative curve describing exploration are generated by program XPLORE.BAS (see Listing 4), a program almost identical to the one just described except that array $\mathrm{M}$ is not cleared after every block of data is printed to the output file and variable CHUNK has a much larger value. These differences produce a cumulative frequency list that, in our case, contains a point for every hour of the session. The resulting ASCII files are used to generate the cumulative exploration curves. These cumulative frequencies can be directly and efficiently compared using the Kolmogorov-Smirnov test (Siegel \& Castellan, 1988).

\section{DISCUSSION}

The basic datum produced by this system consists of a sequence of values representing the status of each of the 48 infrared sensors at the time of sampling. By decoding it, the location of the animal can be determined for every sample. Therefore, if samples were taken several times per second, a reliable digitized account of the animal's location would be obtained, similar to that obtained by the video digitizer detector. Such description would provide fine-grain data on the particular places in the box that the animal visits, the time spent in each of those locations, and the sequence in which the visits occur. When used this way, the system provides a quantitative complement to other types of records, such as film, video tape, or time-lapse photography. Similar data have been obtained by Pear and Eldridge (1984) from video images with a system analogous to those now commer- cially available from Columbus Instruments (Box 44049, Columbus, $\mathrm{OH}$ ), and Coulburn Instruments (Box 2551, Lehigh Valley, PA). These systems support much higher spatial resolutions and are less restrictive with regard to the environments in which they can be used than are the infrared-based alternative presented here, but at a substantially higher cost.

In addition to molecular descriptions of movement, molar characterizations of the animal's location can be produced. A fundamental transformation, used as base for the analyses described above, is the area visited by the animal during a particular time period. On the resulting matrix, a number of partitions and transformations can be performed, yielding various local and global indicators of the spatial distribution of behavior in the chamber. These data allow behavior in the box to be described in reference to either Cartesian or geographical coordinates. Given the sampling rate and spatial resolution afforded by this system, it is possible to effectively use statistical methods to describe molar behavioral adjustments, such as exploration, time allocation, and general activity. A significant feature of the data collected with this method is its simplicity: the raw data merely consist of a sequence of $x, y$ pairs. Therefore, a wide variety of formal manipulations can be validly performed on the basic data and on transformed versions of the data (e.g., area counts). But perhaps more important is the data's apparent adequacy to describe a number of behavioral phenomena. The definitions of exploration, time allocation, and activity used here, while partial to the data, seem to reliably describe a number of orderly events with regard to the spatial distribution of the rat's behavior in the chamber. The system's greater strength derives from its potential application to obtaining functional relations among traditional physiological, ethological, and psychological variables. The chamber can be easily modified to accommodate levers, stimulus lights, feeders, nesting material, and so on. Therefore, studies relating the physical characteristics of the environment to the establishment and maintenance of operant behavior, as well as studies of foraging strategies in patchy environments, can be performed. By adding levels of infrared couples, behavior along the vertical axis, such as rearing and climbing, can be recorded. Also, since the same data describes exploration and general activity, the differential effects of psychotropic drugs (e.g., amphetamine or monoamine oxidase inhibitors) can be directly assessed. Thus far, only a basic set of behaviors have been explored in a very simple physical environment. The system, however, was designed to explore a wide variety of environmental features and a correspondingly complex array of behaviors.

\section{REFERENCES}

Barnett, S. A., \& SMarT, J. L. (1970). Activity of inbred and F1 mice in a residential maze. Quarterly Journal of Experimental Psychology, 22, 494-502.

Brown, R. H. J., \& UNwIN, D. M. (1961). An activity recording system using infra-red detection. Journal of Insect Physiology, 7, 203-209. 
CAMPBELL, B. (1954). Design and reliability of a new activity-recording device. Journal of Comparative \& Physiological Psychology, 47, 90-92.

Dews, P. B. (1953). The measurement of the influence of drugs on voluntary activity in mice. British Journal of Pharmacology, 8, $46-48$.

HaLL, C. S. (1934). Drive and emotionality: Factors associated with adjustment in the rat. Journal of Comparative \& Physiological Psychology, 17, 89-108.

KRNJEVIC, H., VIDEK, M. (1967). A new method of testing CNSactive compounds. Psychopharmacologia, 10, 308-315.

LiVESEY, P. J., LEPPARD, K. (1981). A TV monitored system for recording open-field activity in the rat. Behavior Research Methods, Instruments, \& Computers, 13, 331-333.

Lyons, C. A., \& Cheney, C. D. (1984). Time reallocation in a multiresponse environment: Effects of restricting response classes. Journal of the Experimental Analysis of Behavior, 41, 279-289.

MiLler, T. A. (1979). Insect neurophysiological techniques. New York: Springer-Verlag.

PANKRATZ, A. (1983). Forecasting with univariate Box-Jenkins models. New York: Wiley.

Pear, J. J., E Eldridge, G. D. (1984). The operant-respondent distinction: Future directions. Journal of the Experimental Analysis of Behavior, 42, 453-467.
SHILLITo, E. E. (1970). A method for investigating the effects of drugs on the exploratory behavior of mice. British Journal of Pharmacology, 40, 113-123.

SIEGEL, P. S. (1946). A simple electronic device for the measurement of the gross bodily activity of small animals. Joumal of Psychology, 21, 227-236

Siegel, S., \& Castellan, N. J., JR. (1988). Nonparametric statistics for the behavioral sciences. New York: McGraw-Hill

Silverman, R. W., Chang, A. S., Russell, R. W. (1988). Measurement of activity in small animals using a microcomputer-controlled system. Behavior Research Methods, Instruments, \& Computers, 20 , 537-540.

Staddon, J. E. R., \& Ayers, S. L. (1975). Sequential and temporal properties of behavior induced by a schedule of periodic food delivery. Behaviour, 54, 26-49.

Strong, P. N. (1957). Activity in the white rat as a function of apparatus and hunger. Journal of Comparative \& Physiological Psychology, 50, 596-600.

Ulett, G. A., Heusler, A. Callahan, J. (1961). Objective measurements in psychopharmacology. In E. Rothlin (Ed.), NeuroPsychopharmacology (Vol. 2, pp. 402-409). Amsterdam: Elsevier.

Weasner, M. H., Finger, F. W., \& ReID, L. S. (1960). Activity changes during food deprivation as a function of recording device. Journal of Comparative \& Physiological Psychology, 53, 470-474.

LISTING 1

Program BBSCAN.BAS

MAIN :

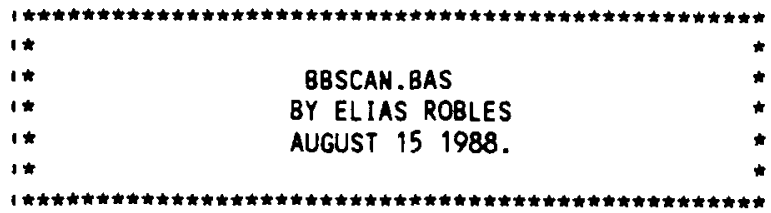

GOSUB SETUP

'Get a sample every $5 \mathrm{sec}$ for the rest of the session ON TIMER(5) GOSUB SCAN

WHILE $N<S L$ : WEND

GOSUB WAYOUT

SETUP:

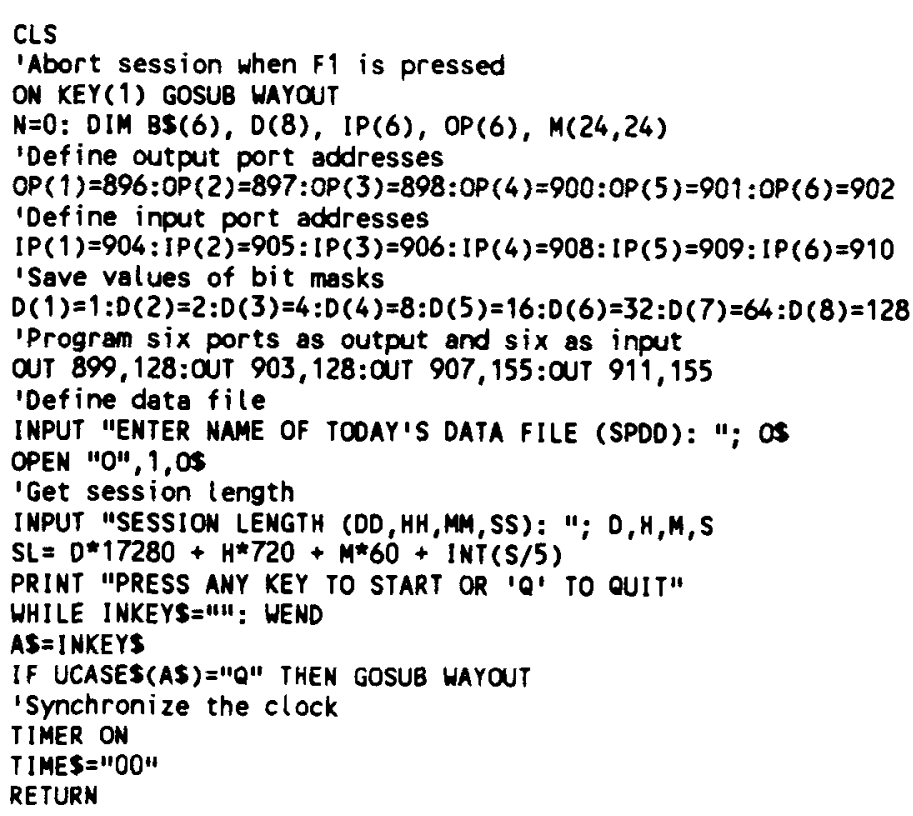


SCAN:

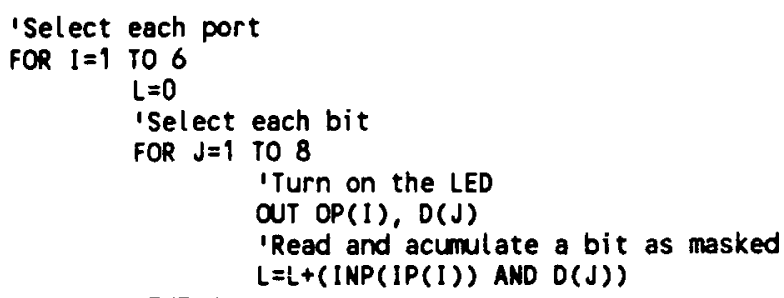

HAYOUT:

- Clear output ports

FOR PORT $=1$ TO 6

NEXT PORT

OUT OP(PORT), 0

TIMER OFF

CLOSE

PRINT "N",N

BEEP (3)

END

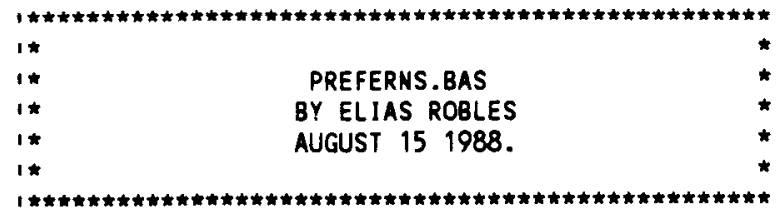

MAIN:

GOSUB SETUP

GOSUB DECOOE

GOSUB GRAPH

GOSUB WAYOUT

SETUP:

CLS

$\operatorname{DIM} M(24,24)$

'Abort when $F 1$ is pressed

ON KEY(1) COSUB WAYOUT

KEY(1) ON

'Def ine $1 / 0$ files

INPUT "ENTER NAME OF INPUT FILE "; Is

INPUT "ENTER NAME OF SURFACE OUTPUT FILE ";O\$

OPEN "I", 1, 1s

OPEN "O", 3, OS

CLS

RETURN 
DECOOE:

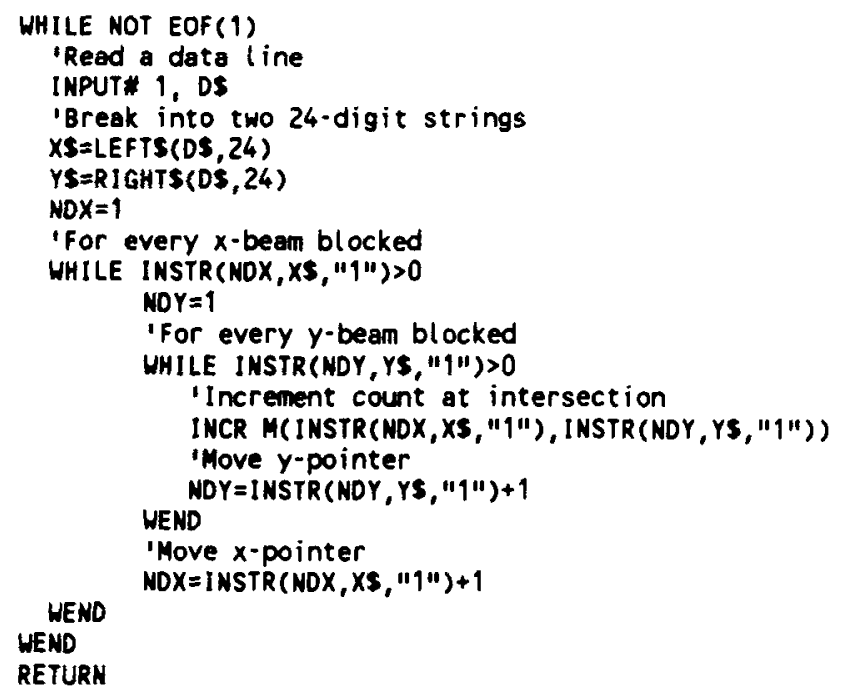

GRAPH :

'Write cumulative frequencies for each $x, y$ pair

$S S=" C: 1 "+0 S+"$. DAT"

OPEN "O", 3, SS

FOR $X=0$ TO 24

FOR $Y=0$ TO 24

NEXT $Y$

PRINT: 3, $X, Y, M(X, Y)$

NEXT $X$

RETURN

WAYOUT:

CLOSE

BEEP (3)

ENO

LISTING 3

Program ACTIVITY.BAS

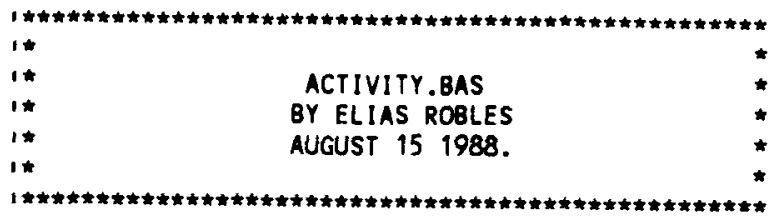

MAIN:

GOSUB SETUP

GOSUB DECOOE

GOSUB HAYOUT

SETUP:

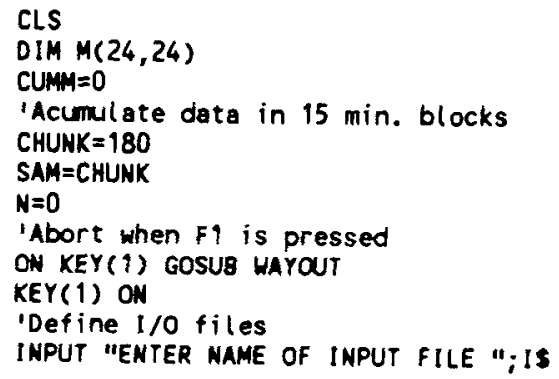


INPUT "ENTER NAME OF SURFACE QUTPUT FILE ";OS

OPEN "I", 1 , IS

OPEN "O",2,OS

CLS

RETURN

DECOOE :

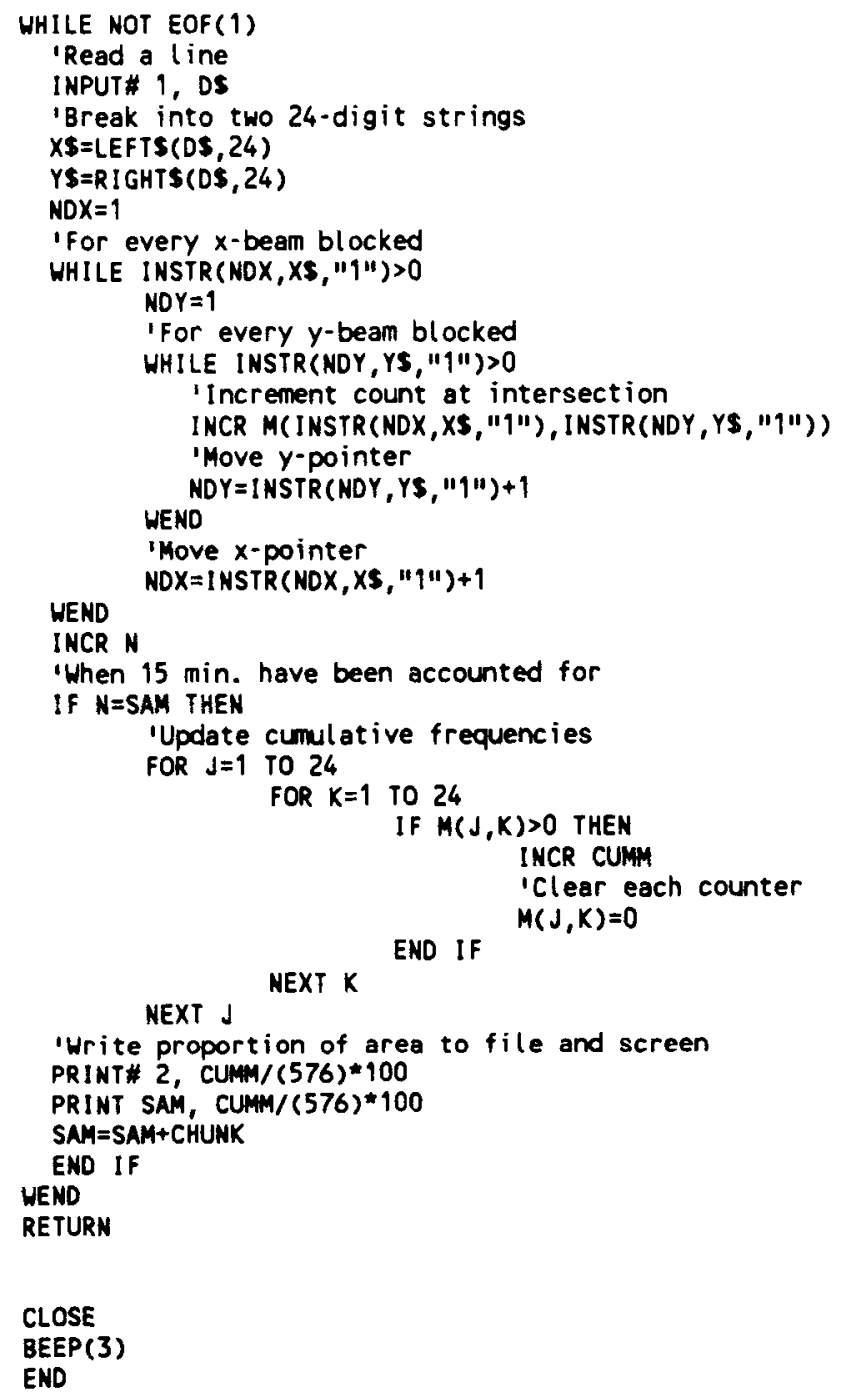

CLOSE

BEEP (3)

END

WAYOUT:

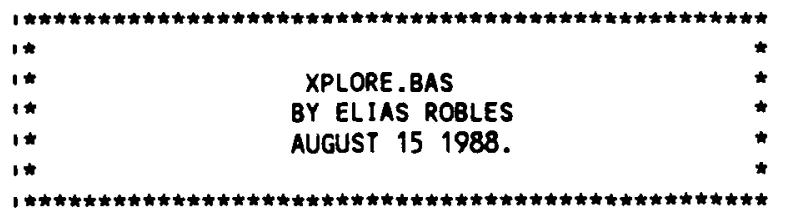

MAIN: 
SETUP:

CLS

$\operatorname{DIM} M(24,24)$

CUMM $=0$

'Number of samples to add

CHUNK $=720$

SAM $=$ CHUNK

$N=0$

'Abort when $F 1$ is pressed

ON KEY(1) GOSUB WAYOUT

KEY(1) ON

'Define $1 / 0$ files

INPUT "ENTER NAME OF INPUT FILE "; Is

INPUT "ENTER NAME OF SURFACE OUTPUT FILE ";OS

OPEN "I", 1, Is

OPEN "O",2, OS

CLS

RETURN

DECOOE:

WHILE NOT EOF(1)

'Read a line

INPUT: 1 , os

'Break into two 24 -digit strings

$X S=$ LEFTS $(0 \$, 24)$

$Y S=R I$ GHTS $(D S, 24)$

NDX $=1$

' For every $x$-beam blocked

WHILE INSTR(NDX, XS, " $1 ")>0$

NDY $=1$

I For every $y$-bean blocked

WHILE INSTR(NDY, YS, "q") >0

I Increment count at intersection

INCR M(INSTR(NDX, X\$, "q"), INSTR (NDY, Y\$, "q"))

iMove y-pointer

NOY=INSTR (NDY, YS, "l")+1

WEND

'Move $x$-pointer

HEND

NOX $=$ I NSTR (NOX, X\$, "I") +1

INCR N

"When an hour has been accounted for

If $N=S A M$ THEN

'Update cumulative frequency data

FOR $J=1$ TO 24

FOR $K=1$ TO 24

NEXT $K$

IF $M(J, K)>0$ THEN INCR CUMM

NEXT J

Write cumulative proportions to file and screen

PRINT* 2, CUNm/(576)*100

PRINT SAM, CUMM/(576)*100

$S A M=S A M+C H U N K$

END IF

WEND

RETURN

WAYOUT :

CLOSE

BEEP (3)

END

(Manuscript received April 20, 1990;

revision accepted for publication October 12, 1990.) 